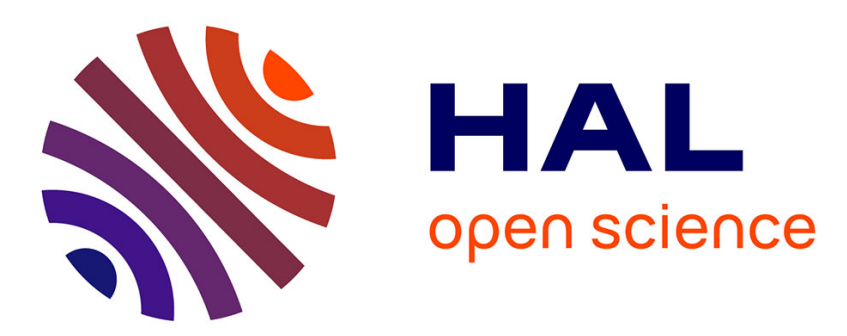

\title{
Teachers' conceptions of nature and environment in 16 countries
}

\author{
François Munoz, Franz Bogner, Pierre Clément, Graça Carvalho
}

\section{To cite this version:}

François Munoz, Franz Bogner, Pierre Clément, Graça Carvalho. Teachers' conceptions of nature and environment in 16 countries. Journal of Environmental Psychology, 2009, 29, pp.407 - 413. 10.1016/j.jenvp.2009.05.007 . hal-01024981

\section{HAL Id: hal-01024981 \\ https://hal.science/hal-01024981}

Submitted on 23 Jul 2014

HAL is a multi-disciplinary open access archive for the deposit and dissemination of scientific research documents, whether they are published or not. The documents may come from teaching and research institutions in France or abroad, or from public or private research centers.
L'archive ouverte pluridisciplinaire HAL, est destinée au dépôt et à la diffusion de documents scientifiques de niveau recherche, publiés ou non, émanant des établissements d'enseignement et de recherche français ou étrangers, des laboratoires publics ou privés. 
Running head: Multivariate analysis of teachers' attitudes

\section{Teachers' conceptions of nature and environment in 16 countries}

François MUNOZ ${ }^{1,4}$, Franz BOGNER ${ }^{2}$, Pierre CLEMENT $^{1}$ and Graça S.CARVALHO ${ }^{3}$

Affiliation: ${ }^{1}$ Laboratoire d'Etude du Phénoméne Scientifique - LIRDHIST (EA 4148),

Université Claude Bernard Lyon 1, 43 Boulevard du 11 Novembre 1918, 69622 Villeurbanne Cedex, France.

${ }^{2}$ University of Bayreuth, Z-MNU (Centre of Maths \& Science Education), D-92455 Bayreuth, Germany.

${ }^{3}$ Institute of Education, University of Minho, Portugal.

${ }^{4}$ UM2, UMR AMAP (botAnique et bioinforMatique de l'Architecture des Plantes), Boulevard de la Lironde, TA A-51/PS2 - F-34398 Montpellier Cedex 5, France.

Corresponding author : François MUNOZ, UM2, UMR AMAP (botAnique et bioinforMatique de l'Architecture des Plantes), Boulevard de la Lironde, TA A-51/PS2, F-34398 Montpellier Cedex 5, France. Tel.: +33 4676175 52. francois.munoz@cirad.fr

\section{Key words}

Conceptions; Attitudes; Nature; Environment; 2-MEV model, Principal component analysis; Between-class analysis; Co-inertia analysis; Orthogonal analysis.

Journal of Environmental Psychology, in press 


\begin{abstract}
By using an original framework involving complementary statistical approaches, we investigated the environmental attitudes of 6,379 pre-service and in-service teachers in 16 countries of Europe and its neighbourhood. To test hypotheses about the nature of environmental attitudes, we examined the variation across groups of individuals (between-class analysis), investigated the independent effects of several candidate explanatory factors (orthogonal analysis), and finally inspected potential relationships between conceptions on a variety of topics (co-inertia analysis).

In accordance with the 2-MEV model of Wiseman and Bogner, we identified two independent dimensions that are, respectively, "Preservation" and "Utilization" oriented. Although attitudes mainly differed across countries with respect to the Utilization dimension, as the influence of socio-economic background seemed to be prominent, the 2-dimensional structure was robust in varying contexts, and teacher discipline was a consistent source of variation in attitudes within countries.
\end{abstract}




\section{Introduction}

Environmental attitudes relate to environmental concerns and may be defined as " the collection of beliefs, affect, and behavioural intentions a person holds regarding environmentally related activities or issues"' (Schultz et al. 2004). A central question is the dimensionality of environmental attitudes (Milfont and Duckitt 2004; Wiseman and Bogner 2003), as thoroughly characterize their structure is important to better predict behaviours (Milfont and Duckitt 2006). We hypothesized EA as a multidimensional construct pertaining to value-based orientations. In a two-dimensional framework (Thompson and Barton 1994), EA may reflect either a concern for all living beings (ecocentric concern) or a concern for humans (anthropocentric concern). In another perspective, Stern (2000) suggested to refer to a threefactor framework of egoistic, altruistic and biospheric concerns. Two issues are to be addressed here, namely, the number of independent dimensions necessary to reliably characterize environmental attitudes, and the nature of the dimensions.

The objective of the European FP6 research project entitled Biohead-Citizen (Carvalho et al. 2004) was to characterize teacher conceptions on a variety of issues, including environmental topics, in order to enhance the quality of education in Europe. In this respect, we devised a questionnaire addressing individual environmental attitudes of pre- and in-service teachers (of Primary school and of Biology and Language secondary schools) in 16 countries. We aimed to test the 2-MEV model (Two Major Environmental Values Model, Wiseman and Bogner 2003), in which two principal value-based orientations, referred to as the Utilization and Preservation dimensions, reflect anthropocentric and ecocentric ecological concerns respectively (Thompson and Barton 1994). We expected a clear discrepancy, and zero correlation, between the Utilization and Preservation dimensions and devised an original statistical framework to explore and test this hypothesis. An alternative result would be, on the other hand, a negative correlation between Utilization and Preservation attitudes, or some more complex pattern.

Apart from characterizing the dimensionality of environmental attitudes, we aimed at understanding the nature of the dimensions. Investigating hypotheses about the effect of underlying social, cultural and economical factors on individual attitudes required addressing many explanatory factors and interpreting complex interactive effects. To such extent, a Multivariate Analysis of Variance (MANOVA) investigates the variation in conceptions across socio-cultural groups, but this often requires meeting many assumptions that are unlikely to be all met simultaneously. To investigate environmental attitudes in the context of our survey, we 
considered a different and somewhat more flexible framework, and to such extent our contribution is methodological and general.

We exposed and discussed the two-dimensional Utilization/Preservation structure on the basis of several hypotheses. We first expected the variation in attitudes across countries to be focused on the Utilization dimension, as an effect of the socio-economic context and country development (2006), and we applied between-class analysis and Monte Carlo test procedures to confirm or infirm this hypothesis, with the advantage of having no MANOVA-like statistical constraints. Conversely, we aimed at testing whether the 2-MEV structure was a by-product of the variation across countries or was instead consistent in different contexts. For this purpose, we used orthogonal analysis to remove the variation across countries and expose the remaining structure.

As the nature of the variation in environmental attitudes may further relate to political positions, according to egoistic and altruistic concerns (Milfont and Gouveia 2006), we further used co-inertia analysis to compare environmental attitudes and political positions. Finally, environmental education is supposed to be a major topic for Primary school teachers and for Biology secondary school teachers. In order to reach reliable recommendations on environmental education throughout 16 countries of the Biohead-Citizen project, we investigated any consistent pattern in teacher attitudes, independently of the variation across countries. Our original results finally shed light upon interesting features and perspectives on environmental psychology and education.

\section{Material and methods}

\subsection{Scope of the research}

We investigated the attitudes of 6,379 pre- and in-service teachers from 16 countries participating in the Biohead-Citizen research project in science education (Carvalho, Clément, $\&$ Bogner, 2004), namely from Cyprus (CY, $n=322$ ), Germany (DE, $n=365)$, Estonia (EE $n$ $=183$ ), Finland (FI, $n=306)$, France (FR, $n=732)$, Hungary (HU, $n=334)$, Italy (IT, $n=$ 559), Lebanon (LB, $n=722$ ), Lithuania (LT $n=316$ ), Malta (MT, $n=198$ ), Morocco (MA, $n$ 330), Poland (PL, $n=311)$, Portugal (PT, $n=351)$, Romania (RO, $n=273)$, Senegal (SN, $n$ $=324$ ) and Tunisia (TN, $n=753$ ), to which we shall henceforth refer using the two-letter ISO codes. Specifically, we used 14 questions to characterize teachers' conceptions towards the nature and the environment (Table 2a). We quantified the answers using a hierarchical, quantitative Likert scale (Likert 1932). 
In each participating country, we applied the questionnaire to a balanced sample of inservice teachers (In) and pre-service teachers (Pre), practicing at Primary schools (P), or teaching Biology (B) or national Language (L) at secondary school, and this yielded six sampling groups (InP, PreP, InB, PreB, InL, PreL). We controlled the sampling to be balanced within each country, while the by-country number of individuals could vary (from 183 in Estonia to 753 in Tunisia), to allow more detailed analyzes in some countries (eg, Lebanon). Teachers filled the questionnaire anonymously in a dedicated room within their school or after a teaching sequence at the University, in the presence of project research fellows. A dedicated section of the questionnaire requested information on demographics (age, gender, etc) and socio-cultural background, including political and religious positions (see summary statistics in Table 1). The overall sample included 4824 (75\%) women and 1555 (25\%) men, whose mean age was 32 , but the sex ratio and age structure varied across countries (Tables $1 \mathrm{a}$ and $1 \mathrm{~b}$ ). The women on men ratio ranged from $16 \%$ in Senegal to $87 \%$ in Poland, while the by-country mean age ranged from 28.6 in Romania to 37.5 in Italy.

\subsection{Characterizing environmental attitudes}

The Model of Ecological Values (2-MEV) postulates that people's positions lay on two orthogonal dimensions, namely, "a biocentric dimension that reflects conservation and protection of the environment (Preservation); and an anthropocentric dimension that reflects the Utilization of natural resources (Utilization)" (Wiseman \& Bogner, (2003). From a similar perspective, Milfont and colleagues (Milfont and Duckitt 2004; Milfont and Gouveia 2006) confirmed the two dimensions showing them "weakly negatively correlated, demonstrating being empirically distinct". Among the 14 questions used to investigate environmental attitudes, 7 related to the Preservation issue, and 7 to the Utilization issue (see wording, labelling and answer coding in Table 2a).

We tested whether teachers' environmental attitudes could reflect this Utilization/Preservation structure, the alternative hypothesis of a single dimensional structure opposing Preservation and Utilization attitudes, or some different picture portraying other trends in attitudes. We used Principal Component Analysis (PCA, see Appendix) to address the issue. This was a first step before assessing the effect of various socio-cultural factors on environmental attitudes. All our statistical analyses were performed by using the ade 4 package within the R software (R Development Core Team, 2006). 


\subsection{Socio-cultural and economic issues relating to environmental attitudes and ecological behaviours}

Using between-class analysis (Appendix §2), we investigated the variation in teachers' environmental attitudes according to their nationality, as it may reflect, at least partly, the influence of socio-cultural and economic backgrounds. Indeed, Milfont and Duckitt (2006) correlated economic liberalism with environmental attitudes pertaining to the Utilization dimension. As our sampling covered a very wide range of economic contexts, we hypothesized the environmental attitudes to mainly vary across countries according to the Utilization dimension. We therefore performed a between-class analysis of the 16 country groups, tested the significance of variation across countries, and finally characterized which questions most differentiated environmental attitudes.

In another perspective, Milfont and Gouveia (2006) investigated the relationship between social conflicts (individual vs. collective interests) and environmental attitudes, and stated that "environmental Preservation [is] positively correlated with future, biospheric, and altruistic [...while] environmental Utilization, on the other hand, [is] positively correlated with selfenhancement, and negatively correlated with future and biospheric" (Milfont \& Gouveia, 2006: p.78). This perspective assumes that ecocentric views (Preservation) relate to more altruistic conceptions, while anthropocentric views (Utilization) relate to more self-centred conceptions. We analyzed a complementary set of "political" questions to investigate the more or less altruistic/selfish respondents' interests. We then correlated teachers' political ideas and their environmental attitudes using co-inertia analysis (Appendix §4).

Environmental attitudes may poorly relate to effective ecological behaviours (Kaiser et al. 1999) and by no means yield a complete picture of teachers' environmental concerns. The impact of environmental attitudes on behaviour is a controversial issue as, for instance, Steel (1996) reported a considerable correlation whereas Diekmann and Preissendörfer (1998) highlighted the discrepancy. We did not monitor individual effective, not even reported, behaviour, but we intended to relate teachers' environmental attitudes with three supplementary questions measuring teachers' declared involvement in environmental matters (Table 2b). We integrated teachers' political ideas (above) and involvement in environmental matters in a single analysis and compared the resulting structure to environmental attitudes using co-inertia analysis (Appendix $\S 4$ ).

\subsection{Environmental attitudes and teaching disciplines}


In order to clarify the potential influence of individual teacher conceptions on the contents of environment education, we investigated the variation in environmental attitudes among our 6 groups of participants: Primary school teachers and pre-teachers and Biology and Language secondary school teachers and pre-teachers. As the wide variation in attitudes across countries may blur the variation across teaching groups, we performed a prior orthogonal Principal Component Analysis controlling for the country effect (Appendix §3) and a subsequent between-class analysis to focus on the country-independent variation in attitudes across teaching groups.

\section{Results}

\subsection{The two-dimensional Utilization/Preservation structure}

We first performed Principal Component Analysis of the questionnaire data and ranked the resulting principal components $\mathbf{C}_{\mathbf{k}}$ according to the proportion of variance they explained. The two first components $\mathbf{C}_{1}$ (abscissa) and $\mathbf{C}_{2}$ (ordinates) represented $15.2 \%$ and $12.5 \%$ of the overall variance of the questions, respectively, and clearly departed in explained variance from the following components. The departure indicated the limit between the two first interpretable and the following trivial components (scree test; Cattell 1966) and suggested that a twodimensional framework was indeed a good basis for investigating teachers' attitudes. Projecting the 14 original questions (arrows) on $\mathbf{C}_{\mathbf{1}}$ and $\mathbf{C}_{2}$ (axes, Figure 1) helped stating the nature of the two dimensions. For each question, the mean answer is at the centre and higher scores are at the tip (score 4 for "I agree"). One group of 7 questions pertaining to Utilization was clearly related to $\mathbf{C}_{\mathbf{1}}$, while the other 7 questions pertaining to Preservation were clearly related to orthogonal $\mathbf{C}_{2}$. To such extent, Utilization attitudes were unrelated to Preservation attitudes. The strong segregation of the 14 questions into two groups gave the significance of components $\mathbf{C}_{1}, \mathbf{C}_{2}$ extra support.

\subsection{Between-country analysis: environmental attitudes across countries}

We then focused on identifying underlying factors that are likely to explain environmental attitudes. We hypothesized that the diversity of social, economic, and cultural contexts across countries may widely explain the variation in attitudes, and indeed the Monte Carlo test of between-country variation was significant $(p$-value $<0.001)$. Furthermore, the first component $\mathbf{D}_{1}$ of the between-country analysis alone explained $63.9 \%$ of the overall variation across countries, and was therefore a major discriminating factor. It clearly related to the Utilization dimension (Pearson's correlation with $\mathbf{C}_{\mathbf{1}}, r=0.91$ ), with a special focus on two most 
discriminating assertions, namely, A16 "Our planet has unlimited natural resources" and A18 "Human beings are more important than other living beings". Moreover, we clearly distinguished on $\mathbf{D}_{\mathbf{1}}$ a group of non-European countries (Morocco, Tunisia, Lebanon and Senegal), with positive values, and a group of European countries, with negative values (Figure 2, abscissa). This suggested a major discrepancy between less and more developed countries with regard to the Utilization dimension. In particular, non-European teachers clearly more agreed with A16 and A18 than European teachers, as they might be more anthropocentric and more confident in natural resource availability. We could hypothesize that countries with a lower economic standard might express less concern about overusing natural resources, as people focus their main concern to meeting their primary needs.

The second between-country component, $\mathbf{D}_{2}$, explained $14.5 \%$ of the overall variation between countries and mainly featured a variation in conceptions across European countries (Figure 2, ordinates). Indeed, more Eastern countries like Lithuania, Poland and Finland clearly deviated on this component, which furthermore strongly related to question A17 (Pearson's $r=$ $0.67, p<0.001$ ), as teachers from these countries agreed more with the sentence "Society will continue to solve even the biggest environmental problems" than teachers from Western European countries. This was a very specific pattern insofar as A17 much more strongly related to $\mathbf{D}_{\mathbf{2}}$ than any other question, of which absolute correlation was always under 0.37 . The position of Lithuania, Poland and Finland on $\mathbf{D}_{\mathbf{1}}$ was furthermore more intermediate regarding the Utilization dimension, hence suggesting that these three Eastern European countries were less concerned by the over-Utilization of environmental resources than Western European countries, as they might be more confident on the ability of the society to overcome the problem.

\subsection{Co-inertia analysis: environmental attitude, political ideas and involvement in environmental matters}

We performed the Principal Component Analysis (PCA, Appendix §1) of political ideas, including (reported) involvement in environmental matters (Table 2b). A subsequent co-inertia analysis allowed addressing the relationship with environmental attitudes (Figure 3). The Monte Carlo permutation test on the co-inertia statistic was clearly significant (p-value $<$ $0.001)$ and, hence, there was a meaningful overall relationship between environmental attitudes, involvement in environmental matters and political ideas. The two first co-inertia axes $\mathbf{C I}_{1}$ and $\mathbf{C I}_{2}$ respectively explained $82.4 \%$ and $10.4 \%$ of the overall co-inertia. The first prominent axis $\mathbf{C} \mathbf{I}_{1}$ clearly related to Utilization questions while $\mathbf{C I}_{2}$ related to Preservation 
ones (Pearson's correlation of $\mathbf{C I}_{\mathbf{1}}$ with $\mathbf{C}_{\mathbf{1}}, r=0.89$, of $\mathbf{C I}_{\mathbf{2}}$ with $\mathbf{C}_{\mathbf{2}}, r=0.86$ ). Moreover, $\mathbf{C I}_{\mathbf{1}}$ linked the Utilization component to political conceptions on economic liberalism, as expressed by the preference for private schools and health systems (PubPriv1, PubPriv2) and for laws favouring the creation of companies (PolitA34) (Figure 3, positive values on abscissa). Furthermore, separation of science from religion or of politics from religion was generally rejected by people with more utilitarian views (Figure 3, PolitA37 and PolitA51 with negative values on the abscissa). This was more present in non-European countries (see Figure 2), mainly Muslim countries (Table 1). On the other hand, PolitA42 and PolitA48 were positively related to $\mathbf{C I}_{1}$, suggesting that less utilitarian people (in European countries) favoured neither a strong central power nor a direct democracy, assigning to the parliament a major political role. The fact that more utilitarian views related to a preference for public pension (low values of PubPriv3) might reflect a cultural difference between European and non-European countries as the former often yield an intermediate answer whereas the latter plainly prefer public pensions.

Among the three questions pertaining to the involvement in environmental matters, and to some extent reporting ecological behaviours, EnvtInv1 was strongly associated with the Preservation co-inertia axis $\mathbf{C I}_{2}$. Despite this relationship, the involvement in association and professional contexts was shown to be poorly related to either the Preservation or the Utilization components. This might confirm that the link between ecological behaviours and environmental attitudes is not straightforward, and that the nature of reported involvement indeed matters.

\subsection{Orthogonal analysis: environmental attitudes across teaching groups when controlling for the country effect}

Using orthogonal analysis, we extracted two prominent orthogonal components $\mathbf{C}_{1}{ }_{1}$ and $\mathbf{C}_{2}$ that did not depict any variation between countries, but still pertained to the Utilization and Preservation value-based orientations (Pearson's correlations with $\mathbf{C}_{\mathbf{1}}$ and $\mathbf{C}_{\mathbf{2}}$ after varimax rotation, respectively, $r=0.96$ and $r=0.97$ ). This meant that the structure of environmental attitudes yielded by the basic Principal Component Analysis (Figure 1) reflected not only variation across countries (Figure 2) but also revealed the same pattern on average within the 16 countries. Hence the 2-dimensional structure of environmental attitudes was consistent and transversal to a variety of socio-cultural contexts.

Using the Monte Carlo permutation test, we further showed a very significant countryindependent variation in environmental attitudes across teaching groups ( $p$-value $<0.001$ ). Some questions differentiated strongly between teaching groups (Figure 4): A16 ("our planet 
has unlimited natural resources") and A18 ("human beings are more important than other living beings") contributed mostly to differentiate Biology teachers (InB and PreB) from other groups, as they disagreed preferentially with this statement. Interestingly, A18 mostly differentiated PreB from other groups whereas A16 mostly differentiated InB from other groups, suggesting some divergence correlated with the training level in Biology. Furthermore, A40 ("it is interesting to know what kinds of animals live in ponds or rivers") contributed mostly to differentiate Language teachers ( $\mathrm{InL}$ and PreL), who in general more disagreed with this statement. Attitudes of national Language teachers were on the whole less naturalistic than other teachers, independently of any country-dependent context.

\section{Discussion}

We have investigated the structure of pre- and in-service teachers' environmental attitudes throughout 16 countries, using a far broader sampling than most previous studies. We have found a two-dimensional Preservation/Utilization structure supporting the 2-MEV hypothesis (Bogner et al. 2000; Bogner and Wiseman 1997; Bogner and Wiseman 1999). This structure proved to be a robust reference for characterizing the variation in attitudes according to a variety of factors, such as country and teaching discipline, but also religion (not shown), and hence appeared to be consistent and general. Apart from the two-dimensional structure, the fact that the Utilization and Preservation components are orthogonal (as shown in Figure 1 and 3) points to the fact that they portray independent conceptions, that is, teachers' Utilization attitudes are unrelated to their Preservation attitudes. This is really different, for instance, from an alternative picture with Preservation and Utilization attitudes opposing along a single component, as teachers' opinions regarding resource Utilization would be then negatively correlated with their opinion regarding nature Preservation.

On the other hand, the variance explained by $\mathbf{C}_{\mathbf{1}}$ and $\mathbf{C}_{\mathbf{2}}$ summed to less than $30 \%$, which is not high regarding the number of questions, 14 , but which is substantial regarding the number of individuals, 6379. A substantial part of the variance may be explained by secondary, yet still not significant, components featuring contingent sources of variation from a so large number of questionnaires. Apart from the main trend in Utilization and Preservation attitudes, there was place for variation among individuals from any fixed "stereotype" of answers. This would be contingent upon individual contexts, while $\mathbf{C}_{1}$ and $\mathbf{C}_{2}$ might catch a structure of more general value and hence are relevant features.

The value-belief-norm theory of Stern (2000) advocates, on the other hand, a three-factor structure according to egoistic, altruistic and biospheric orientations. This puts focus on 
concerns about adverse consequences (AC) of environmental degradation or protection, insofar as it can affect the fate of human beings (altruistic concern), of the individual (egoistic concern), or of species in general (biospheric concern). Although this framework was first proposed for characterizing behaviours, this is still a relevant basis for characterizing attitudes (see Snelgar 2006). But this is not, in principle, contradictory to a two-dimensional UtilizationPreservation structure, insofar as both frameworks do not address the same issues. People favouring resource Utilization can have egoistic as well as altruistic concerns, as well as people favouring nature Preservation can. Hence the three factors of the value-belief-norm theory can be located in a two-dimensional structure as well, as it does not imply the three factors to be independent components. Independence of the Utilization and Preservation dimensions is on the other hand a key result helping disentangle environmental attitudes, without prejudice of the underlying concerns. We could design another questionnaire to more precisely characterize the nature of the concerns, and this is indeed a perspective.

We have further demonstrated the influence of local socio-economic contexts, as our results have highlighted that European and non-European teachers' conceptions vary with regard to the Utilization dimension above all. Moreover, the co-inertia analysis yielded a Utilization-oriented variation across countries that can be related to the variation in political views regarding economic liberalism, as suggested by Milfont \& Duckitt (Milfont and Duckitt 2006). Furthermore, after orthogonal analysis removing between-country variation, the Utilization-Preservation dimensions were still prominent, demonstrating that this structure is not a pure by-product of a variation across countries. Another interesting result is the attitude variation across teaching groups: this was still significant when the variation between countries was statistically removed and points to the more or less naturalistic conceptions of teachers according to the discipline.

A crucial and open point is the relationship between environmental attitudes and related (reported) behaviours. We focused on teachers' declared personal involvement in environmental matters at three levels, namely, locally (family, friends), in associations, and in a professional context. Using co-inertia analysis, we have shown that only the local involvement relates well to Preservation attitudes. Thus, further research is needed to characterize the complex pattern of environmental behaviours in order to examine this controversial relationship (Bateson and Ma 1999). 
This work demonstrates how using a variety of multivariate statistical procedures (details in Appendix) is of broad interest in the field of science education, and, more generally, in the fields of human and social sciences (see also Tacq 1997). The proposed framework indeed allows calculating synthetic components as indices of a person's conceptions, helps investigating the significance of any variation in conceptions between groups of individuals (by using between-class analysis), exposes the separate effects of concurrent causal factors (using orthogonal analysis), and exposes the links between conceptions on different issues (using co-inertia analysis). One may use sampling groups based upon nationality, teaching subject, or religion, as well as featuring any other socio-cultural question of interest. On the whole, between-class and orthogonal analyzes provide valuable alternatives to MANOVA and MANCOVA parametric approaches, especially when the use of such tests is prevented by a clear violation of the assumptions involved.

Investigating environmental attitudes in 16 European countries and neighbouring countries has yielded fruitful results and an original discussion of theoretical expectations, and this may open up new perspectives in the field of environmental psychology. Furthermore, the proposed statistical approach is a promising tool for studies of other topics based upon the BioheadCitizen questionnaire, such as health education and sex education, as well as for other related studies relying on questionnaires in social sciences.

\section{Acknowledgements}

This research was founded by the European project FP6 BIOHEAD-CITIZEN CIT2-CT-2004506015 (Biology, Health and Environmental Education for better Citizenship). We especially thank all the project's participants who gathered all data in their countries. We also thank P. Couteron, S. Holmes, B. Oerke and M. Wiseman and for their helpful comments on the manuscript.

\section{References}

Bateson, D. J., and Ma, X. (1999). "A Multivariate Analysis of the Relationship Between Attitude Toward Science and Attitude Toward the Environment." Journal of Environmental Education

Bogner, F. X., Brengelmann, J. C., and Wiseman, M. (2000). "Risk -taking and environmental perception." The Environmentalist, 20, 49-62.

Bogner, F. X., and Wiseman, M. (1997). "Environmental perception of rural and urban pupils." Journal of Environmental Psychology, 17, 111-122. 
Bogner, F. X., and Wiseman, M. (1999). "Toward measuring adolescent environmental perception." European Psychologist, 4, 139-151.

Carvalho, G., Clément, P., and Bogner, F. (2004). "Biology, Health and Environmental Education for better Citizenship, STREP CIT2-CT-2004-506015, E.C., Brussels, FP6, Priority 7." STREP CIT2-CT-2004-506015, E.C., Brussels, FP6, Priority 7.

Cattell, R. B. (1966). "The scree test for the number of factors." Journal of Multivariate Behavioral Research, 1, 245-276.

Diekmann, A., and Preissendörfer, P. (1998). "Environmental behavior: discrepancies between aspirations and reality." Rationality and Society, 10(1), 79-102.

Kaiser, F. G., Wölfing, S., and Fuhler, U. (1999). "Environmental attitude and ecological behaviour." Journal of Environmental Psychology, 19, 1-19.

Likert, R. (1932). "A Technique for the Measurement of Attitudes." Archives of Psychology, $140,1-55$.

Milfont, T. L., and Duckitt, J. (2004). "The structure of environmental attitudes: A first- and second-order confirmatory factor analysis." Journal of Environmental Psychology, 24, 289-303.

Milfont, T. L., and Duckitt, J. (2006). "Preservation and Utilization: Understanding the Structure of Environmental Attitudes." Medio Ambiente y Comportamiento Humano, 7(1), 29-50.

Milfont, T. L., and Gouveia, V. V. (2006). "Time perspective and values: An exploratory study of their relations to environmental attitudes " Journal of Environmental Psychology, 26(1), 72-82.

R_Development_Core_Team.(2006). R: A language and environment for statistical computing, R Foundation for Statistical Computing, Vienna, Austria.

Schultz, P. W., Shriver, C., Tabanico, J. J., and Khazian, A. M. (2004). "Implicit connections with nature." Journal of Environmental Psychology, 24, 31-42.

Snelgar, R. S. (2006). "Egoistic, altruistic, and biospheric environmental concerns: Measurement and structure." Journal of Environmental Psychology, 26(2), 87-99.

Steel, B. S. (1996). "Thinking globally and acting locally ? : Environmental attitudes, behavior and activism." Journal of Environmental Management, 47(1), 27-36.

Stern, P. (2000). "Toward a coherent theory of environmentally significant behavior." Journal of social issues, 56(3), 407-424.

Tacq, J. (1997). Multivariate analysis techniques in social science research: from problem to analysis, Sage publication, London. 
Thompson, S. C. G., and Barton, M. A. (1994). "Ecocentric and anthropocentric attitudes toward the environment." Journal of Environmental Psychology, 14, 149-157.

Wiseman, M., and Bogner, F. X. (2003). "A higher-order model of ecological values and its relationship to personality." Personality and Individual Differences, 34, 783-794.

Table 1: Information on sampled individuals throughout the 16 countries.

Labels of countries: CY (Cyprus), DE (Germany), EE (Estonia), FI (Finland), FR (France), HU (Hungary), IT (Italy), LB (Lebanon), LT (Lithuania), MA (Morocco), MT (Malta), Poland (PL), PT (Portugal), RO (Romania), SN (Senegal), TN (Tunisia).

(a) Male and female balance (lines) in countries (columns)

\begin{tabular}{ccccccccccccccccc}
\hline & CY & DE & EE & FI & FR & HU & IT & LB & LT & MA & MT & PL & PT & RO & SN & TN \\
\hline F & 251 & 252 & 153 & 256 & 557 & 271 & 497 & 593 & 294 & 191 & 164 & 290 & 287 & 237 & 90 & 441 \\
M & 71 & 113 & 30 & 50 & 175 & 63 & 62 & 129 & 22 & 139 & 34 & 21 & 64 & 36 & 234 & 312 \\
\hline
\end{tabular}

(b) Age structure (lines) in countries (columns)

\begin{tabular}{lllllllllllllllll}
\hline & CY & DE & EE & FI & FR & HU & IT & LB & LT & MA & MT & PL & PT & RO & SN & TN \\
\hline-20 & 27 & 12 & 7 & 36 & 1 & 50 & 0 & 9 & 34 & 49 & 11 & 0 & 11 & 114 & 3 & 13 \\
$(20,25]$ & 75 & 146 & 91 & 102 & 302 & 111 & 108 & 255 & 115 & 110 & 63 & 158 & 159 & 16 & 51 & 350 \\
$(25,30]$ & 131 & 40 & 13 & 39 & 117 & 51 & 122 & 110 & 12 & 26 & 39 & 31 & 32 & 35 & 110 & 124 \\
$(30,35]$ & 42 & 25 & 11 & 16 & 78 & 25 & 73 & 144 & 20 & 13 & 26 & 14 & 18 & 37 & 33 & 69 \\
$(35,40]$ & 11 & 30 & 7 & 33 & 64 & 16 & 43 & 103 & 35 & 19 & 31 & 29 & 18 & 40 & 37 & 72 \\
$(40,45]$ & 12 & 21 & 21 & 19 & 49 & 26 & 29 & 41 & 34 & 46 & 4 & 39 & 33 & 10 & 31 & 74 \\
$(45,50]$ & 18 & 22 & 14 & 19 & 61 & 30 & 62 & 34 & 37 & 47 & 6 & 30 & 48 & 8 & 38 & 28 \\
+50 & 6 & 69 & 19 & 41 & 60 & 25 & 122 & 26 & 29 & 20 & 18 & 10 & 32 & 13 & 21 & 23 \\
\hline
\end{tabular}

(c) Individuals in countries (columns) and religion groups (lines)

\begin{tabular}{ccccccccccccccccc}
\hline & CY & DE & EE & FI & FR & HU & IT & LB & LT & MA & MT & PL & PT & RO & SN & TN \\
\hline AGN & 13 & 49 & 80 & 46 & 370 & 51 & 69 & 3 & 13 & 2 & 1 & 6 & 33 & 20 & 3 & 14 \\
CHR & 285 & 280 & 52 & 221 & 304 & 221 & 444 & 217 & 288 & 1 & 193 & 295 & 296 & 250 & 27 & 1 \\
ELS & 24 & 35 & 50 & 39 & 47 & 62 & 46 & 33 & 15 & 6 & 4 & 10 & 22 & 3 & 5 & 15 \\
MUS & 0 & 1 & 1 & 0 & 11 & 0 & 0 & 469 & 0 & 321 & 0 & 0 & 0 & 0 & 289 & 723 \\
\hline
\end{tabular}


Table 2: Questions on environmental issues fall into two categories: questions pertaining to environmental attitudes (a) and to political ideas and behaviours (b). In most cases, responses to the questions are coded using four levels, from disagree (1) to agree (4). Details on specific coding are provided when needed.

(a) Questions featuring environmental attitudes in the Biohead-Citizen questionnaire.

Questions fall into two groups, P (Preservation) and U (Utilization).

\begin{tabular}{ll}
\hline Cod & Question \\
\hline A1 (P) & We must set aside areas to protect endangered species. \\
A4 (U) & Nature is always able to restore itself. \\
A5 (P) & If an intensive chicken farm were going to be created near where you live, \\
& you would be against this because it may pollute the groundwater. \\
A7 (P) & Humans will die out if we don't live in harmony with nature. \\
A11 (P) & Industrial smoke from chimneys makes me angry. \\
A16 (U) & Our planet has unlimited natural resources. \\
A17 (U) & Society will continue to solve even the biggest environmental problems. \\
A18 (U) & Human beings are more important than other living beings. \\
A22 (P) & I enjoy trips to the countryside. \\
A23 (U) & We need to clear forests to increase agricultural areas. \\
A32 (U) & Humans have the right to change nature as they see fit. \\
A40 (P) & It is interesting to know what kinds of animals live in ponds or rivers. \\
A50 (P) & All contemporary plant species should be preserved because they may help \\
& in the discovery of new medicines. \\
A54 (U) & Only plants and animals of economic importance need to be protected. \\
\hline
\end{tabular}

(b) Questions featuring political ideas and ecological behaviours in the Biohead questionnaire

Cod Question

Questions on political ideas in the main section on the questionnaire

PolitA15 A priority of the government must be to guarantee resources for health protection of the poor.

PolitA20 My government should compel all immigrants to learn to speak, to read and to write in (my state language). 
PolitA26 There are too many foreigners in my country: the government should limit immigration.

PolitA34 The government must make laws favouring the creation of firms to stimulate our economy.

PolitA37 Religion and politics should be separated.

PolitA42 Only a strong central power can put some order in my country.

PolitA48 Direct democracy (without government involvement) is the ideal solution to managing our society.

PolitA51 Science and religion should be separated.

Questions concerning involvement in environmental matters in personal information section

Specific coding of the answers: Are you involved in activities pertaining to environmental conservation and/or sustainable development? (Tick only ONE box in EACH line; Never = cod 1 , Sometimes $=\operatorname{cod} 2$, Often $=\operatorname{cod} 3)$.

EnvtInv1 At home, in your family, in your local community.

EnvtInv2 In an organisation (formal or informal).

EnvtInv3 Professionally.

Questions concerning public and private institutions in personal information section

Specific coding of the answers: Which institution you trust more: Public or Private? (Tick

ONE box in EACH line)

PubPriv1 Public schools (cod 1) to Private schools ( $\operatorname{cod} 4)$.

PubPriv2 Public health services $(\operatorname{cod} 1)$ to Private health services $(\operatorname{cod} 4)$.

PubPriv3 Public pension ( $\operatorname{cod} 1)$ to Private pension $(\operatorname{cod} 4)$. 


\section{Figure captions}

Figure 1: Picture of the relationship between the two main Principal Components and the 14 questions measuring teachers' environmental attitudes. Abscissa and ordinates are respectively the first principal component, $\mathbf{C}_{\mathbf{1}}$, explaining $15.0 \%$ of the overall variance, and the second principal component, $\mathbf{C}_{2}$, explaining $12.5 \%$ of the overall variance. Arrows reflect the 14 original questions using the labels provided in Table 2a. Projecting a question onto abscissa or ordinates shows its contribution to the axis, and helps characterize the value-based orientations underlying the principal components. Projecting an arrow onto another reflects the partial correlation between the two questions in $\left(\mathbf{C}_{\mathbf{1}}, \mathbf{C}_{2}\right)$. Two closed arrows show a high degree of correlation in the $\left(\mathbf{C}_{\mathbf{1}}, \mathbf{C}_{\mathbf{2}}\right)$ space, for example A54 and A23, which are almost superimposed.

Figure 2: Between-country analysis of the 14 questions measuring the teachers' environmental attitudes. We show the position of country groups of individuals, which are labelled using twoletter ISO cods, according to the most differentiating components $\left(\mathbf{D}_{\mathbf{1}}, \mathbf{D}_{\mathbf{2}}\right)$. This highlights on $\mathbf{D}_{1}(63.9 \%$ of the between-country variance) an overall contrast between non-European countries (negative values) and European countries (positive values), and on $\mathbf{D}_{\mathbf{2}}$ (14.5\% of the variance) the contrast between a group of three countries (Lithuania, Finland and Poland) and the others.

Figure 3: Co-inertia analysis of the 14 questions measuring the teachers' environmental attitudes, on one hand, and the 14 questions measuring their political ideas and involvement in environmental matters, on the other hand. We display the political ideas according to the two first co-inertia axes $\mathbf{C I}_{\mathbf{1}}$ (abscissa) and $\mathbf{C I}_{2}$ (ordinates), which pertain to the Utilization and Preservation dimensions.

Figure 4: Between-teaching group analysis with control of the variation between countries, yielding the ranked between-class components $\mathbf{D}_{\mathbf{k}}$. The centroids of the teaching groups are

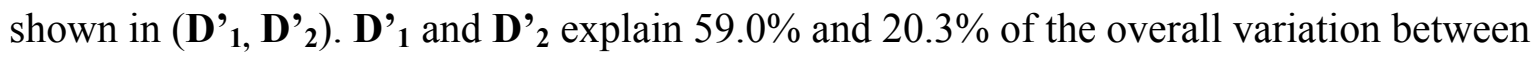
teaching groups, respectively. 
Figure 1

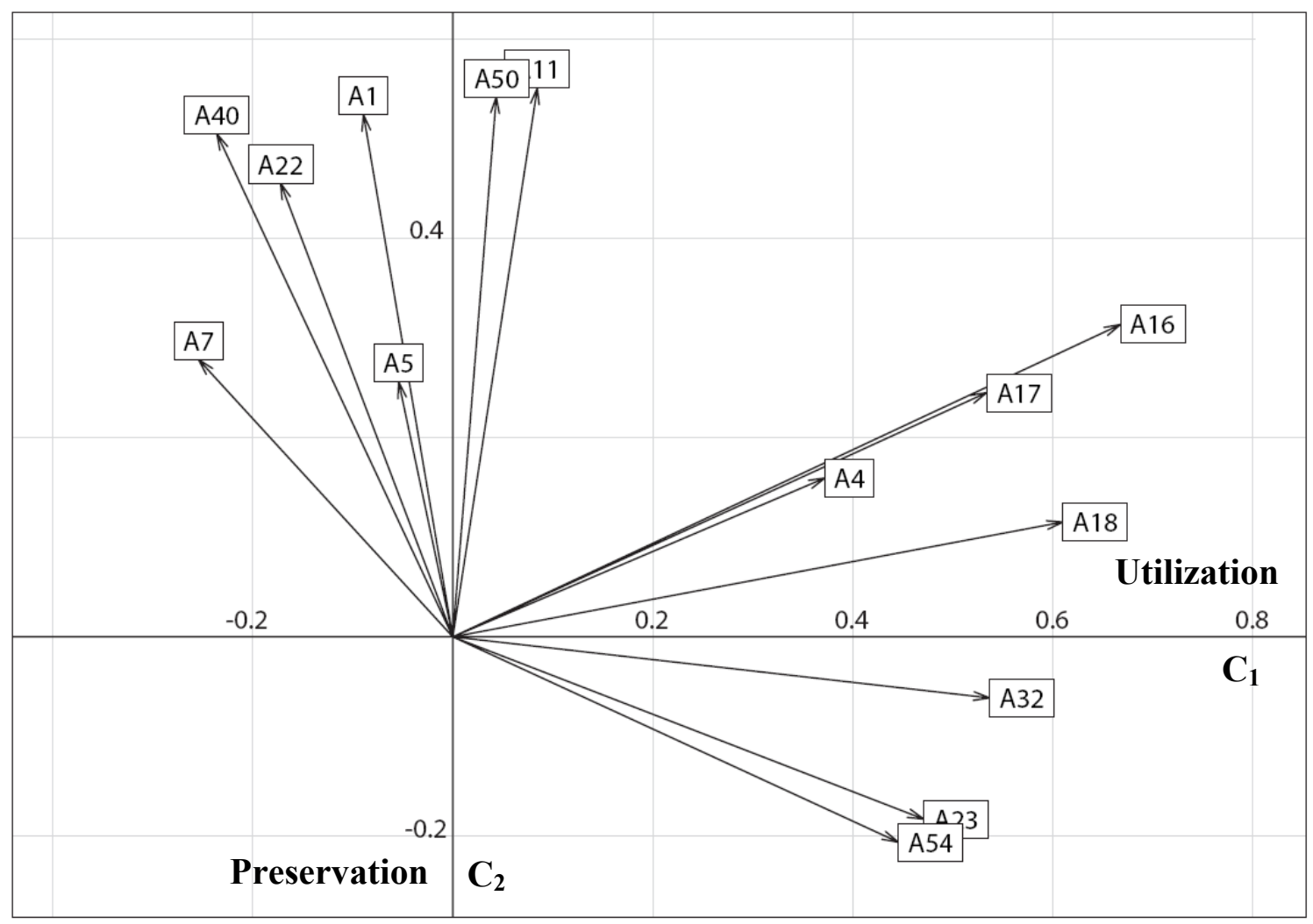


Figure 2

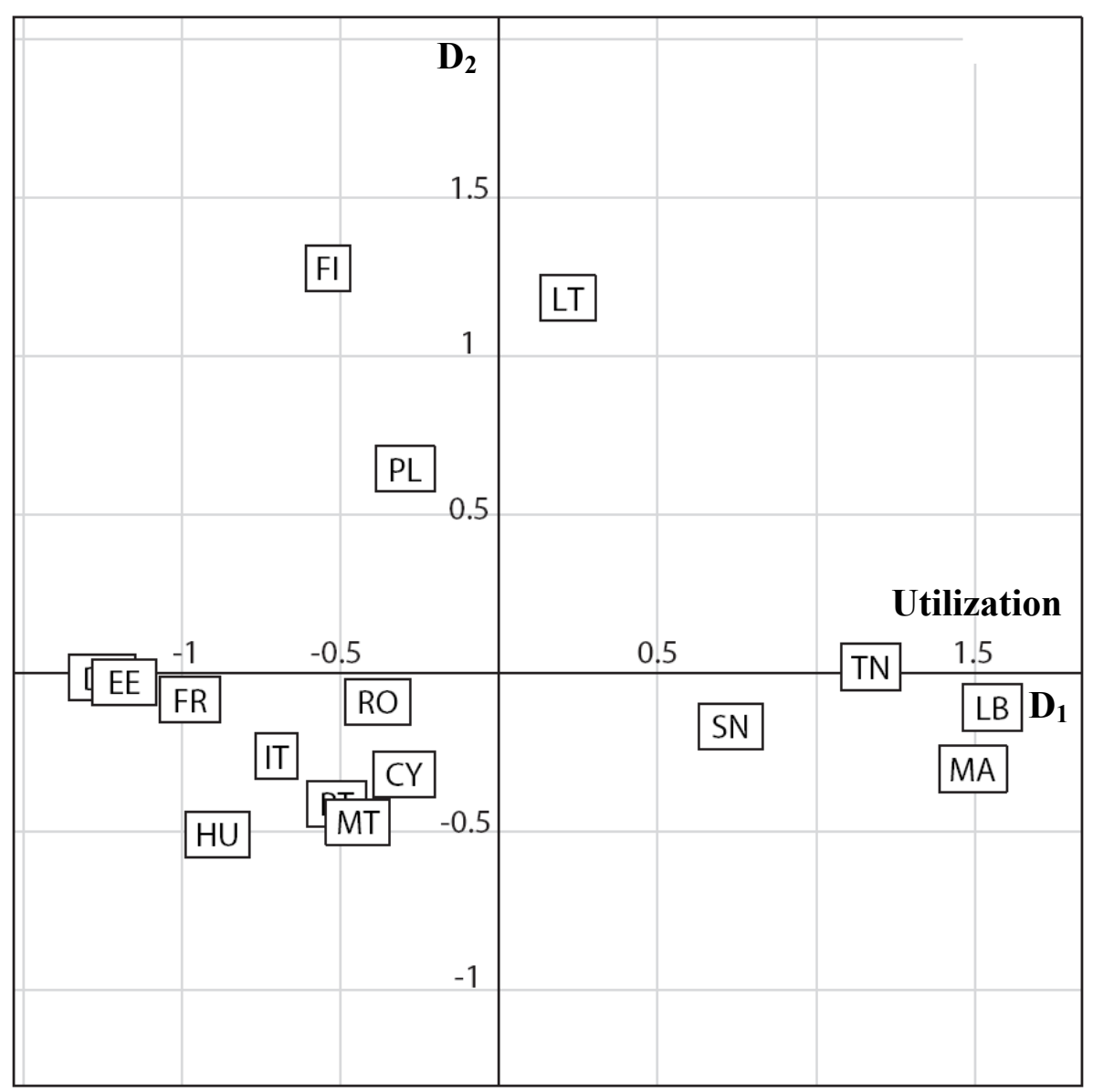


Figure 3

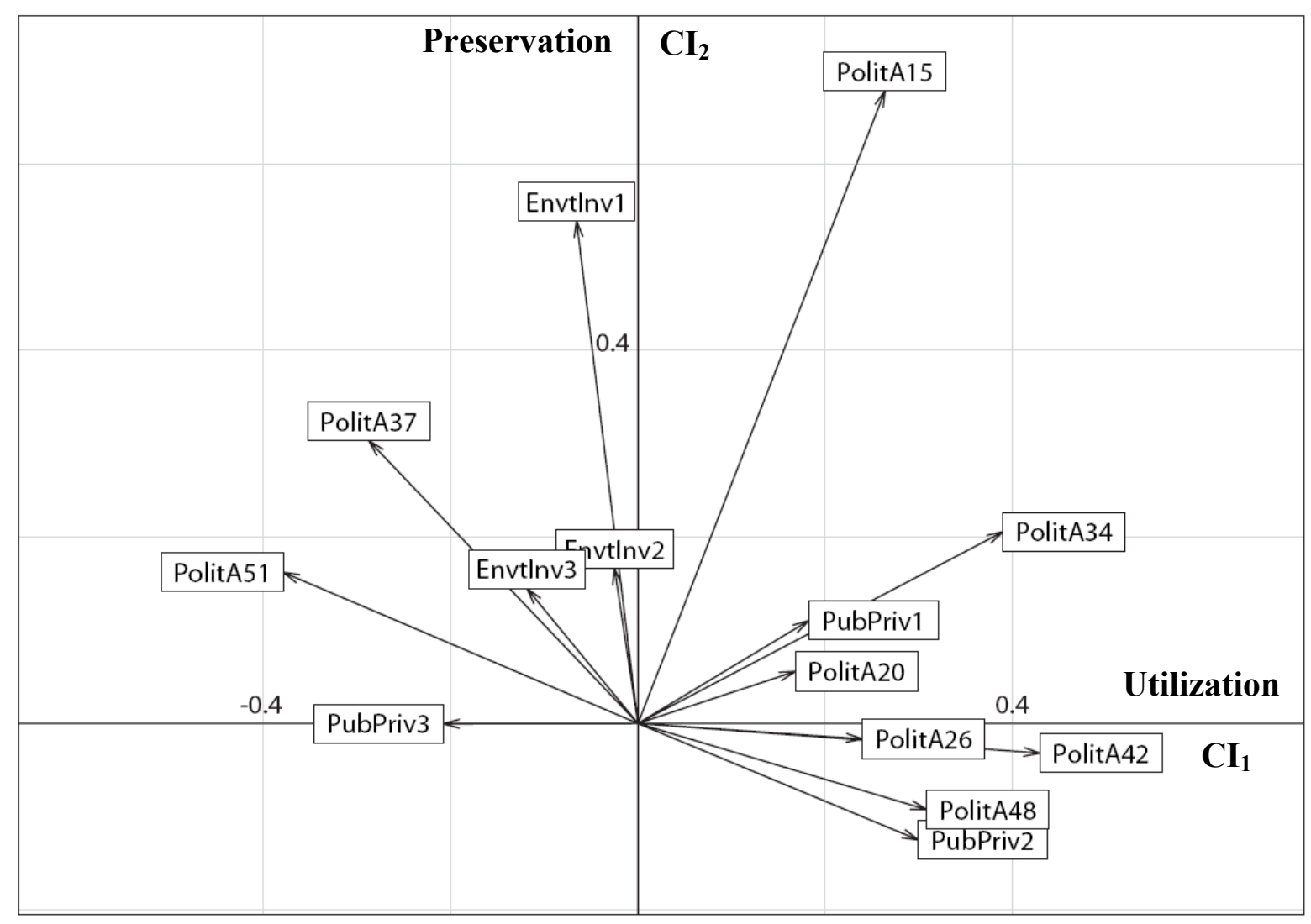


Figure 4

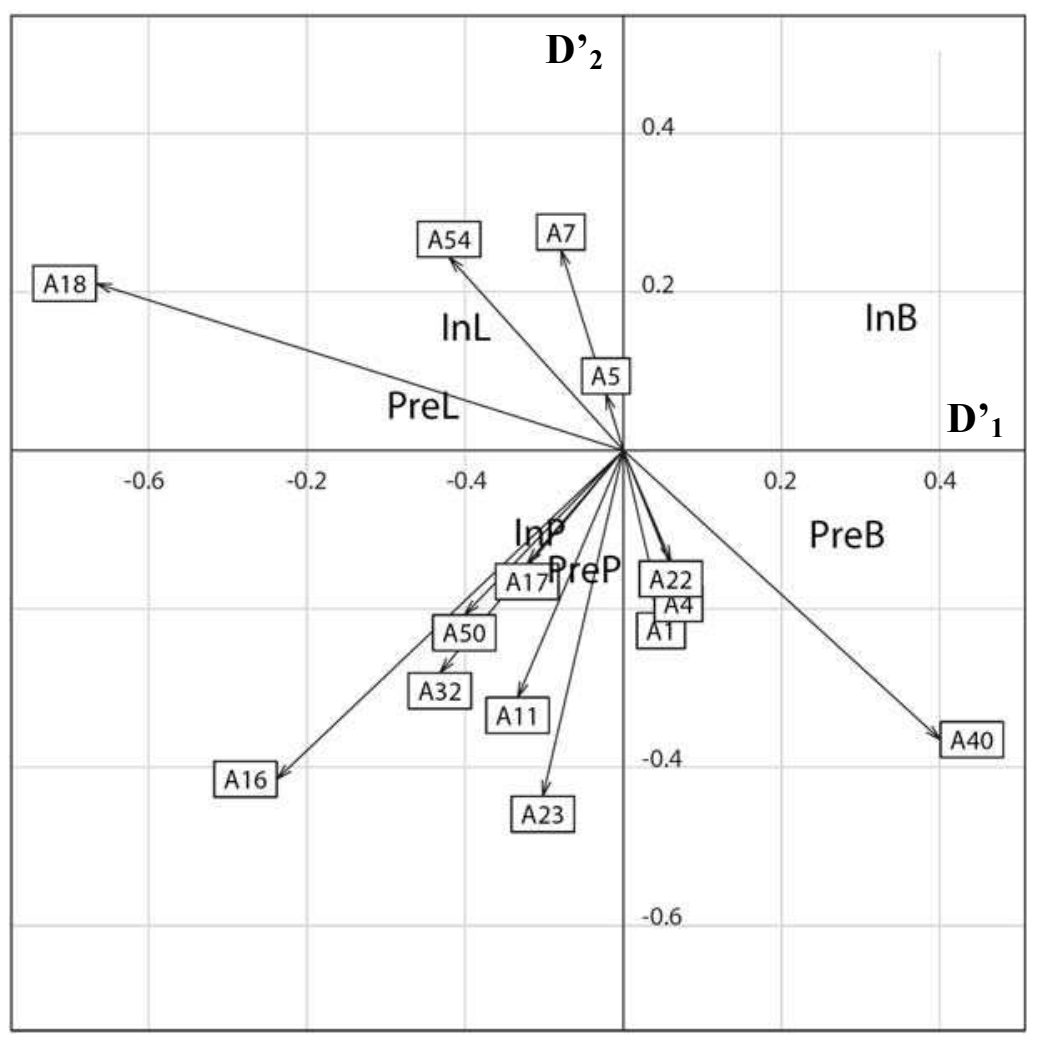




\section{Online appendix: statistical guidelines}

The Appendix material first emphasizes $(\S 1)$ the core principle of a multivariate analysis of variance - covariance, which allows characterizing the variation in individual conceptions with regard to specific research concerns in science education. Then we expose how to use between-class analysis and an adequate permutation test (Good, 2005) to assess whether conceptions differ significantly between sub-samples, in order to extract significant predictors (§2), orthogonal analysis to separate the partially independent effects of alternative but possibly correlated predictors $(\S 3)$, co-inertia analysis to compare the conceptions merging from separate multivariate data sets and investigate whether they feature convergent systems (§4). We refer to relevant functions from the package ade4 (see Thioulouse, Chessel, Dolédec, \& Olivier, 1997) of the R statistical software (R Development Core Team, 2006), throughout the methodological exposé.

\section{Multivariate analysis: the starting point}

Let us consider a set of $n$ individuals $i$ answering to $p<n$ questions $j$ enclose in a questionnaire. In the sequel, the question $\mathbf{X}_{\mathbf{j}}$ will be a standardized column vector, that is, with mean 0 and variance 1 over individuals $i$, of the answers for question $j$, and the entire table will be noted $\mathbf{X}$. The $p \times p$ matrix of variance-covariance $\mathbf{V}=\mathbf{X}^{\prime} \cdot \mathbf{X}$ features, at position $(i, j)$, with $i$ $\neq j$, the covariance of $\mathbf{X}_{\mathbf{i}}$ and $\mathbf{X}_{\mathbf{j}}$, while elements on the diagonal contain the variance of the $p$ questions, here equal to 1 because questions are standardized. One may perform specific weighting or rescaling operations on the rows and columns of $\mathbf{X}$, to feature other kinds of variance - covariance matrices. One eventually investigates the overall structure of $\mathbf{X}$ through the eigen-analysis of the symmetric matrix $\mathbf{V}$, with only 1 s on the diagonal in our case. The eigen-decomposition of $\mathbf{V}$ provides positive or null eigenvalues $\lambda_{k}$ and corresponding eigenvectors $\mathbf{Z}_{\mathbf{k}}$. Finally, the principal components $\mathbf{C}_{\mathbf{k}}$ are linear combinations of the primary questions $\mathbf{X}_{\mathbf{j}}$ through the relationship $\mathbf{C}_{\mathbf{k}}=\mathbf{X} . \mathbf{Z}_{\mathbf{k}}$. The principal components are orthogonal, independent questions, and hence usually reflect independent features from the answers to a questionnaire. The covariance between principal components is therefore null, and one can sort them according to decreasing variance, i.e., according to decreasing corresponding eigenvalues. This exemplifies the basic case of Principal Component Analysis (PCA, function dudi.pca), but a wide array of multivariate approaches is now available, according to varying preferred designs of the variance - covariance matrix.

Principal Component Analysis is dedicated to quantitative data, that is, in the context of questionnaire data, to responses that are numerically ordered. If the responses are instead 
qualitative, using PCA in prevented from and one should use Correspondence Analysis (CA). Although the mathematics is somewhat different, CA likewise helps stating the main trends in conceptions arising from the qualitative responses to a questionnaire. According to the nature of the data, one would thereby use either PCA or CA to first characterize the main features of the data, before applying the following tools to address further specific questions.

\section{Between-class analysis}

The $n$ individuals may belong to several sampling groups that can reflect, in science education, different socio-cultural backgrounds. An important issue is to address whether conceptions differ between groups, and we suggest using between-class analysis for this purpose (between function) as it provides independent between-class components $\mathbf{D}_{\mathbf{k}}$ that are ranked according to the between-class variance they depict. This is in fact a particular kind of multivariate analysis with respect to instrumental variables (see Lebreton, Sabatier, Banco, \& Bacou, 1991), where there is only one instrumental, factor variable. One then relates $\mathbf{D}_{\mathbf{k}}$ to the primary questions $\mathbf{X}_{\mathbf{j}}$, and thus characterizes the conceptions that most differ between the groups according to the answers to the questionnaire (e.g. main text Figure $4 \mathrm{~b}$ for teaching groups).

To some extent, this framework relates to the one-way MANOVA, in which sampling groups classically represent the levels of a treatment. One may test whether there is any significant difference in conceptions between groups using a non-parametric permutation test. A Monte Carlo procedure allows iteratively and randomly reallocating the group labels to individuals, and hence provides the probability distribution of the overall between-class inertia, under the null hypothesis of no variation between groups (using randtest function family, specifically randtest.between for between-class analysis).

\section{Orthogonal analysis}

The between-class analysis allows testing the effect of one categorical factor (sampling groups) on people conceptions, as MANOVA does. One may repeat the analysis to separately test the effect of other categorical factors, yet without including the effect of possibly misleading interactions. Following the principle of variance partitioning, one may alternatively test the effect of a factor independently of the effect of one or several covariates, using orthogonal analysis (pcaivortho function). This analysis provides some "orthogonal" principal components, that is, components that are designed to be algebraically orthogonal, hence statistically independent, from a set of covariates. This allows separating the variance of people 
conceptions that is related to the covariates, and the variance that is unrelated to them.

Eventually, one can perform a between-class analysis (between function) according to another categorical factor of interest, in order to test its effect when controlling for the effects of the ruled-out covariates.

\section{Co-inertia analysis}

It may be worth investigating separately the structure of several multivariate data sets in order to examine specific features from each analysis. One may further question whether the conceptions pertaining to the separate issues are interdependent, such as through any common socio-cultural and economic background. In the field of ecology, one may likewise seek to separately elucidate the structure of species abundances and the information on environmental factors, and subsequently assess their common structure characterizing the species ecological requirements along the community gradients (Dolédec \& Chessel, 1994; Dray, Chessel, \& Thioulouse, 2003). Then the optimising criterion is that the scores of observations in both tables should be the most covariant ones.

The analysis provides co-inertia axes that can be related to the questions or to corresponding principal components from each of the two multivariate data sets. We rank and select the axes according to their eigenvalues, and then characterize the co-inertia axes using question coordinates. A Monte Carlo procedure based on the overall co-inertia is available in the package ade4 and allows testing whether the co-inertia between the two multivariate data sets significantly deviate from chance (function randtest.coinertia).

\section{References of the Online appendix}

Dolédec, S., and Chessel, D. (1994). Co-inertia analysis: an alternative method for studying species-environment relationships. Freshwater Biology, 31(3), 277-294.

Dray, S., Chessel, D., and Thioulouse, J. (2003). Co-inertia analysis and the linking of ecological tables. Ecology, 84(11), 3078-3089.

Good, P. (2005). Permutation, Parametric, and Bootstrap Tests of Hypotheses - A Practical Guide to Resampling Methods for Testing Hypotheses. In Springer series in statistics (3rd edition). New York: Springer.

Lebreton, J. D., Sabatier, R., Banco, G., and Bacou, A. M. (1991). Principal component and correspondence analyses with respect to instrumental variables : an overview of their role in studies of structure-activity and species- environment relationships. In .J. 
Devillers, \& W. Karcher (Eds), Applied Multivariate Analysis in SAR and Environmental Studies (pp. 85-114). Dordrecht: Kluwer Academic Publishers.

R Development Core Team (2006). R: A language and environment for statistical computing, Vienna: R Foundation for Statistical Computing.

Thioulouse, J., Chessel, D., Dolédec, S., and Olivier, J. M. (1997). ADE-4: a multivariate analysis and graphical display software. Statistics and Computing, 7, 75-83. 